\title{
NEW ESTIMATIONS OF THE REMAINDER IN THREE-POINT QUADRATURE FORMULAE OF EULER TYPE
}

\section{Klaričić Bakula, J. Pečarić, M. Ribičić Penava And A. Vukelić}

Abstract. We derive some new bounds for the general three-point quadrature formulae of Euler type using some inequalities for the Chebyshev functional. As special cases, we provide some new error estimates for Euler Simpson formula, dual Euler Simpson formula and Euler Maclaurin formula. Also, applications for Euler Bullen-Simpson formula are obtained.

Mathematics subject classification (2010): 26D15, 26D20, 26D99.

Keywords and phrases: Chebyshev functional, Three-point quadrature, Euler Simpson formula, dual Euler Simpson formula, Euler Maclaurin formula, Euler Bullen-Simpson formula.

\section{REFERENCES}

[1] M. Abramowitz AND I. A. STEgun (Eds), Handbook of Mathematical Functions with Formulas, Graphs, and Mathematical Tables, National Bureau of Standards, Applied Mathematics Series 55, 9th printing, Washington, 1970.

[2] A. Aglić Aljinović, A. ČIvljak, S. Kovač, J. PeČArić, M. Ribičić Penava, General Integral Identities and Related Inequalities, Element, Zagreb, 2013.

[3] P. S. Bullen, Error estimates for some elementary quadrature rules, Univ. Beograd Publ. Elektrotehn. Fak. Ser. Mat. Fiz., 602-633 (1978), 97-103.

[4] P. Cerone, S. S. Dragomir, Some new Ostrowski-type bounds for the Čebyšev functional and applications, J. Math. Inequal. 8, 1 (2014), 159-170.

[5] I. Franjić, J. PeČArić, I. Perić, Quadrature formulae of Gauss type based on Euler identities, Math. Comput. Modelling 45, 3-4 (2007), 355-370.

[6] I. Franjić, J. PeČArić, I. Perić, A. Vukelić, Euler integral identity, quadrature formulae and error estimations, Element, Zagreb, 2011.

[7] M. Matić, J. Pečarić, A. Vukelić, On generalization of Bullen-Simpson's inequality, Rocky Mountain J. Math. 35 (2005), 1727-1754.

[8] D. S. Mitrinović, J. E. PeČArić, A. M. FinK, Classical and New inequalities in Analysis, Kluwer Academic, Dordrecht, 1993.

[9] B. G. PAChPATte, On Čebyšev-Grüss type inequalities via Pečarić's extension of the Montgomery identity, J. Inequal. Pure Appl. Math. 8, 1 (2006), Article 11, 4 pp. (electronic).

[10] J. PeČARIĆ, On the Čebyšev inequality, Bul. Inst. Politehn. Timisoara 39, 25 (1980), 10-11. 